\title{
Seroprevalence of Zoonotic Parasites in Pigs Slaughtered in the Kathmandu Valley of Nepal
}

\author{
Brecht Devleesschauwer,,2 Mathieu Pruvot, ${ }^{3}$ Durga Datt Joshi, ${ }^{4}$ Stéphane De Craeye, ${ }^{5}$ Malgorzata Jennes, \\ Anita Ale, ${ }_{4}^{4}$ Alma Welinski, ${ }^{6}$ Sanjyoti Lama, ${ }^{4}$ Arjun Aryal, ${ }^{7}$ Bjorn Victor, ${ }^{8}$ Luc Duchateau, ${ }^{9}$ Niko Speybroeck, ${ }^{2}$ \\ Jozef Vercruysse, and Pierre Dorny ${ }^{1,8}$
}

\begin{abstract}
For several years, the demand for pork has been on the rise in Nepal. To assess the importance of pork as a carrier of zoonotic agents, we performed a cross-sectional study in the Kathmandu Valley of Nepal, in which we serologically determined the infection status of slaughtered pigs with regard to three of the most important parasites transmitted through pork consumption: Trichinella spp., Taenia solium cysticerci, and Toxoplasma gondii. From 2007 to 2010, 742 pigs were sampled at slaughter, of which $0.1 \%$ (95\% confidence interval [CI] $0.0-0.7 \%$ ) were found positive for Trichinella infection, $13.8 \%$ (95\% credibility interval [CrI] $0.8-28.5 \%$ ) for T. solium cysticercosis, and $11.7 \%$ (95\% CI 5.2-17.5\%) for Toxoplasma infection. Further monitoring of the related animal and human disease burden and strengthening of food safety protocols throughout the pork production chain are strongly recommended.
\end{abstract}

Key Words: Nepal—Pigs—Seroprevalence-Taenia solium-Toxoplasma gondii_Trichinella spp.

\section{Introduction}

$\mathbf{N}$ ePAl is a South Asian country, situated between India and China, with a population of over 27 million people. In recent years, the country has seen remarkable progress in overall development (Tropp 2009), which is reflected in an increasing rate of urbanization and a growing middle class. As a consequence, the demand for animal protein is increasing, particularly in the urban centers of the country, such as the Kathmandu Valley, the economic and political heart of the country. Associated with the weakening of social barriers against pork consumption, the pig sector, which is geographically clustered in the Kathmandu Valley, the southern Terai lowlands, and eastern Nepal, has become one of the fastest growing livestock sectors in Nepal. The pig population, which stood at 592,000 in 1990, grew to 913,000 in
2000 (FAO/UNDP 2003), and has reached 1,108,465 according to the latest data from 2010/2011 (DLS 2011). The gross production of pork has also shown a strong increase, but its share in the total meat production of the country remains constant at over $7 \%$, due to the general increase in meat production (FAO 2010).

Pig production and slaughtering systems, however, have not evolved at the same pace as pig population growth. Pigs are typically raised extensively or semiextensively, although more and more professional pig farmers are converting to a complete intensive system. Over the last 50 years, exotic breeds such as Landrace, New Hampshire, and Yorkshire are being farmed in addition to the local breeds (Bampudke, Chwanche, and Hurra). A local crossbreed, the Dharane Kalo Bangur or Pakhribas Black, is currently as popular as the foreign breeds, especially in the eastern part of Nepal

\footnotetext{
${ }^{1}$ Department of Virology, Parasitology and Immunology, Faculty of Veterinary Medicine, Ghent University, Merelbeke, Belgium.

${ }^{2}$ Institute of Health and Society, Université catholique de Louvain, Brussels, Belgium.

${ }^{3}$ Department of Production Animal Health, Faculty of Veterinary Medicine, University of Calgary, Calgary, Alberta, Canada.

${ }^{4}$ National Zoonoses and Food Hygiene Research Centre, Tahachal, Kathmandu, Nepal.

${ }^{5}$ Scientific Institute of Public Health, Communicable and Infectious Diseases, National Reference Center for Toxoplasmosis, Brussels, Belgium.

${ }^{6}$ Ecole Nationale Vétérinaire de Toulouse, Toulouse, France.

${ }^{7}$ Central Veterinary Hospital, Tripureshwor, Kathmandu, Nepal.

${ }^{8}$ Department of Biomedical Sciences, Institute of Tropical Medicine, Antwerp, Belgium.

${ }^{9}$ Department of Comparative Physiology and Biometrics, Faculty of Veterinary Medicine, Ghent University, Merelbeke, Belgium.
} 
(Kayastha 2006, Sharma 2006). Due to the absence of professional pig slaughterhouses, pigs are generally slaughtered on slaughter slabs or in the streets. According to the Animal Slaughterhouse and Meat Inspection Act, all slaughtered animals and meat should be examined by a meat inspector. However, the Government of Nepal has so far been unable to implement these regulations (Joshi et al. 2003).

Given the increased demand for pork and the disproportionately slow progress of production systems, the importance of pork as a carrier of zoonotic agents is likely to increase. The consumption of raw or poorly cooked pork can be a source of various zoonotic diseases, including parasitic zoonoses such as trichinellosis, taeniosis, and toxoplasmosis (Dorny et al. 2009). Assessing and monitoring the potential threat emerging from this growing market will therefore become of increasing importance for safeguarding public health. To this aim, we performed a cross-sectional study in slaughterhouses of the Kathmandu Valley, Nepal, in which we serologically determined the infection status of slaughtered pigs with regard to three of the most important parasites transmitted through pork consumption: Trichinella spp., Taenia solium cysticerci, and Toxoplasma gondii.

\section{Materials and Methods}

\section{Study area}

This study was conducted in the Kathmandu Valley of Nepal. A total of 16 slaughter slabs were included in this study, spread over five distinct areas of the Kathmandu Valley, i.e., Talchhikhel, Jadibuti, Sinamangal, Teku, and Tahachal. Because there is no list of existing slaughter slabs in the Kathmandu Valley, these slabs were identified based on local knowledge and snowball sampling, an iterative nonprobability sampling where study participants suggest more participants for recruitment.

\section{Serum sampling}

Blood samples were collected at slaughter. A numbered plastic tube was filled with $10 \mathrm{~mL}$ of blood from the posterior vena cava or vena porta after removal of the liver. The blood tubes were transferred to the laboratory of the National Zoonoses and Food Hygiene Research Centre (Kathmandu, Nepal), and, after clotting overnight at $4^{\circ} \mathrm{C}$, centrifuged for $15 \mathrm{~min}$ at $1800 \times \mathrm{g}$. The serum was then transferred to $1.5-\mathrm{mL}$ microcentrifuge tubes and stored at $-20^{\circ} \mathrm{C}$ until analysis. Samples were also spotted on Whatman filter paper (two $40-\mu \mathrm{L}$ spots and two $10-\mu \mathrm{L}$ spots), incubated in a hot-air oven at $60^{\circ} \mathrm{C}$ for $1 \mathrm{~h}$, air-dried, and stored at $4^{\circ} \mathrm{C}$ until transfer to the Institute of Tropical Medicine (ITM), Antwerp, Belgium, where they were analyzed (Wilmaerts 2010).

A total of 822 samples were collected between 2007 and 2010, of which 742 could be screened for all three parasites. For the sake of comparability, seroprevalences were estimated based on the latter set of samples. The reported breeds and origins could be grouped in three categories, i.e., White (exotic) pigs originating from the Kathmandu Valley and adjoining districts $(n=335)$, Pakhribas Black originating from the southeast of Nepal $(n=333)$, and Hurra originating from the wider south of Nepal and the north of India $(n=74)$. The average age of the sampled animals was 8.5 months (range 3$36)$, and the overall male-to-female ratio was 4.8 .

\section{Serological methods}

Trichinella spp. An enzyme-linked immunosorbent assay (ELISA) was used to screen the serum samples for the presence of immunoglobulin $\mathrm{G}(\mathrm{IgG})$ antibodies $(\mathrm{Ab})$ against Trichinella spp. excretory/secretory (ES) antigens (Vercammen et al. 2002). The samples were tested at the ITM, Antwerp, Belgium, after eluting the spotted filter papers in blocking buffer (i.e., phosphate-buffered saline containing $0.05 \%$ Tween 20 and $2 \%$ newborn calf serum). Previous experiments have shown that storage and transport on filter papers does not affect the results of the Trichinella spp. ES AbELISA (Wilmaerts 2010), and other authors have successfully applied this method (Thi et al. 2013). Samples were tested in duplicate and were considered positive if their mean optical density (OD) exceeded a plate-specific cutoff level, which was determined as the 99.9 th percentile of a modified Student $t$ distribution fitted to the mean and standard deviation of eight negative control samples (Frey et al. 1998). The latter were obtained from naïve Belgian animals.

Although the Trichinella spp. ES Ab-ELISA is considered a highly sensitive test, its specificity is often unsatisfactory. Gómez-Morales et al. (2008), for instance, reported a sensitivity of $>99 \%$, and a specificity of $90.6 \%$. Positive and doubtful samples were therefore confirmed by an ES-antigenbased western blot (Yera et al., 2003) at the ITM (Antwerp, Belgium), and reconfirmed, using the same test, at the European Union Reference Laboratory for Parasites (Rome, Italy). A recent study has defined a three-band pattern, ranging in size from 48 to $72 \mathrm{kD}$ as being distinctive for Trichinella infection in pigs. Further evaluations showed that this distinctive pattern has a sensitivity and specificity of $100 \%$ (Gómez-Morales et al. 2012).

T. solium cysticerci. Serum samples were tested at the National Zoonoses and Food Hygiene Research Centre, Kathmandu, Nepal, for the presence of circulating Taenia spp. cysticercal ES antigens, using the B158/B60 Ag-ELISA described by Dorny et al. (2004). This test shows a good performance for the detection of viable Taenia spp. cysticerci, but is not able to differentiate the different porcine Taenia species, i.e., T. solium, T. hydatigena, and T. asiatica (Deckers and Dorny 2010). Samples were tested in duplicate and results were reported following the same method as outlined above.

Because no confirmation test was available to assess the true infection status, an estimate of the true prevalence was obtained by combining the apparent prevalence with prior information on the test characteristics in a Bayesian model (Speybroeck et al., 2013). Two cases were considered, i.e., the performance of the test for detecting viable Taenia spp. cysticerci, and the performance for detecting T. solium cysticerci. In the first case, test sensitivity was modeled as a Uniform $(0.60$, $1.00)$ distribution, and test specificity as a Uniform $(0.90,1.00)$ distribution (Dorny et al. 2004). In the second case, the specificity was modeled as a Uniform distribution ranging from $75 \%$ to $100 \%$, reflecting the potentially high proportion of positive test results due to infection with T. hydatigena or $T$. asiatica, which are both known to occur in Nepal (Devleesschauwer et al. 2012). This Bayesian model was fitted in OpenBUGS (Lunn et al. 2009), using the BRugs package (Thomas et al. 2006) in $\mathrm{R}$ version 2.15.0 (R Development Core Team 2012). 
T. gondii. The serum samples were tested for the presence of IgG antibodies against the T. gondii SAG1 surface antigen using the ELISA protocol described by De Craeye et al. (2008). All samples were tested in duplicate at the ITM, Antwerp, Belgium, after elution of the filter papers in blocking buffer. Because we had no information on the T. gondii SAG1 ELISA test characteristics in our setting and we were not able to confirm positive samples, we applied the prevalence assessment method proposed by Opsteegh et al. (2010). First, the natural logarithm of the mean OD was calculated for each sample. Then, a binormal mixture distribution was fitted to these results using the mixdist package for $\mathrm{R}$ (Macdonald and Du 2011), thereby providing two overlapping normal distributions with different means and variances. The distribution with the lower mean represents the population of negative samples, whereas the distribution with the higher mean represents the population of positive samples. The ratio of the area under the curve of the normal distribution with the higher mean to that of the overall distribution was therefore considered as an estimate of the apparent Toxoplasma infection prevalence (Opsteegh et al. 2010). To obtain a 95\% confidence interval (CI) for the prevalence, the results of the 742 samples were resampled with replacement, and a binormal mixture distribution was fitted to each bootstrap sample. A 95\% CI was then constructed by taking the $2.5^{\text {th }}$ and $97.5^{\text {th }}$ percentile of the simulated prevalences. This procedure was based on 1000 bootstrap samples and was performed in $\mathrm{R}$ version 2.15.0.

To evaluate the effect of spotting, storing, and transporting the serum samples on filter paper, a subset of the samples was also tested using the modified agglutination test (MAT; Desmonts and Remington 1980). Correspondence between ELISA and MAT results was assessed via Spearman rank correlation coefficient.

\section{Results}

The resulting seroprevalence estimates for the different zoonotic parasites are summarized in Table 1. Eighty out of the 742 serum samples tested positive on the Trichinella spp. Ab-ELISA (10.8\% [95\% exact CI 8.6-13.2\%]). One of these positive responders showed the distinctive three-band pattern as defined by Gómez-Morales et al. (2012) on both western blots, resulting in a Trichinella infection prevalence of $0.1 \%$ (95\% exact CI $0.0-0.7 \%$ ). The positive animal was a 9month-old male White pig sampled in 2010.

One hundred forty-two samples tested positive on the Taenia spp. Ag-ELISA (19.1\% [95\% exact CI 16.4-22.2\%]).

\section{Table 1. Seroprevalence Estimates of Zoonotic Parasites in Slaughter Pigs Sampled in the Kathmandu Valley, Nepal, BETWEEN 2007 AND $2010(N=742)$}

\begin{tabular}{lrc}
\hline & \multicolumn{2}{c}{ Estimated seroprevalence } \\
\cline { 2 - 3 } Parasite & mean & 95\% Uncertainty interval (\%) \\
\hline Trichinella spp. & $0.1 \%$ & $0.0-0.7$ \\
Taenia spp. cysticerci & $19.7 \%$ & $11.1-30.0$ \\
Taenia solium cysticerci & $13.8 \%$ & $0.8-28.5$ \\
Toxoplasma gondii & $11.7 \%$ & $5.2-17.5$ \\
\hline
\end{tabular}

Bayesian posterior estimates of true prevalence were $19.7 \%$ (95\% credibility interval [CrI] 11.1-30.0\%) for Taenia spp. cysticercosis, and $13.8 \%$ (95\% CrI $0.8-28.5 \%)$ for T. solium cysticercosis.

On the basis of the T. gondii SAG1 ELISA test results, the binormal mixture model estimated an apparent Toxoplasma infection prevalence of $11.7 \%$ (Fig. 1). The bootstrap method yielded a $95 \%$ CI ranging from $5.2 \%$ to $17.5 \%$. Twenty-four samples were subjected to MAT, and the resulting titers were in good correspondence with the ELISA OD values (Spearman rho = 0.75), indicating that the ELISA was not knowingly disturbed by the pretreatment of the samples.

\section{Discussion}

In this cross-sectional study, we determined the seroprevalence of three zoonotic parasites in slaughtered pigs in the Kathmandu Valley of Nepal. In accordance with previous studies (Joshi et al. 2001, Joshi et al. 2005, Sapkota et al. 2006, Karn et al. 2008), we found a very low Trichinella infection prevalence of $0.1 \%$ and a true $T$. solium infection prevalence ranging from $0.8 \%$ to $28.5 \%$. In addition, we assessed the prevalence of porcine Toxoplasma infection in Nepal, which ranged from $5.2 \%$ to $17.5 \%$. This result significantly adds to the few information available on the $T$. gondii infection status in pigs in Nepal, as the only studies published on this topic so far were either not current (Rai et al. 1996) or based on a limited number of animals (Datt Bhatt 2012).

The serological tests that were applied in this study are indicative of current infections with the three considered parasites, and these results may therefore be seen as a proxy

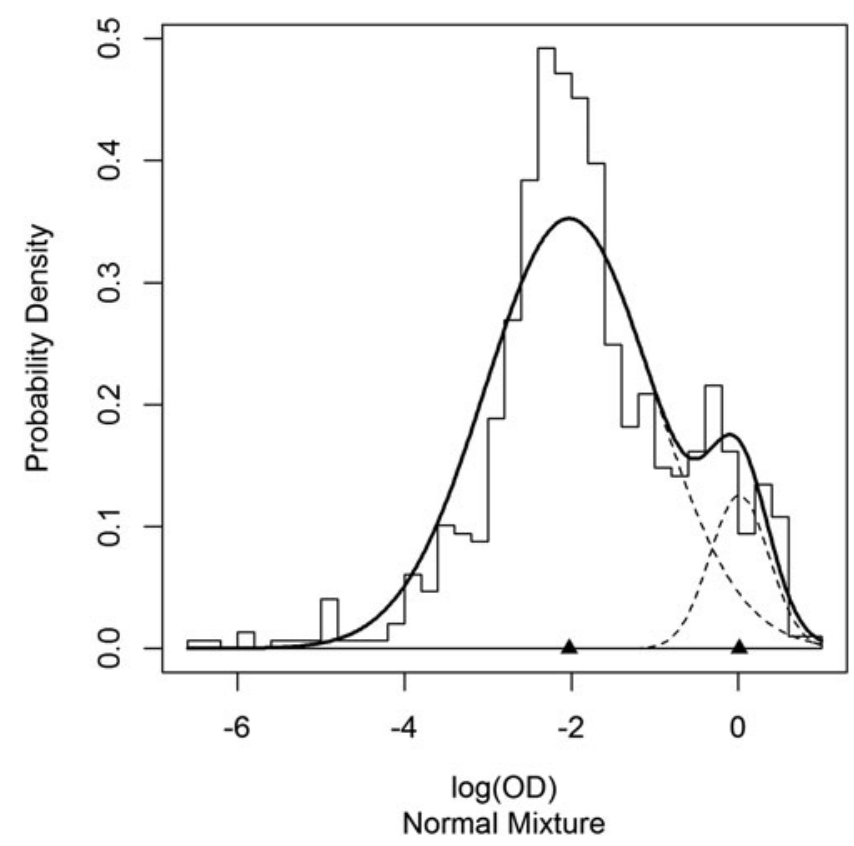

FIG. 1. Binormal mixture model fitted to the natural logarithm of the mean optical densities (ODs) of the T. gondii SAG1 Ab-ELISA. (stepped line) histogram of $\log (\mathrm{OD})$; (solid bold curve) fitted binormal mixture distribution; (dashed thin curves) fitted individual normal distributions; (triangles) means of fitted individual normal distributions. This graph was produced with the mixdist package in $\mathrm{R}$. 
for the risk of infection when consuming raw or undercooked pork. Indeed, circulating Taenia spp. cysticercal antigens represent current infections with viable cysticerci, whereas anti-Taenia spp. antibodies reflect exposure, but not necessarily current infection (Praet et al. 2010). Aborted infections seem to be a common phenomenon in the transmission dynamics of Taenia spp. (Devleesschauwer et al. 2013), whereas animals infected with Trichinella spp. or T. gondii are believed to remain positive throughout their life. The presence of antibodies against the latter two parasites may therefore be regarded as an indication of current infection.

Due to the nature of the serological tools we used, we could only obtain an estimate of the apparent infection prevalence. However, by performing confirmation tests, where possible, and using advanced statistical techniques, where needed, we believe that this study provides a good insight into what may be the true prevalence of these infections.

In addition, although snowball sampling has the advantage of easily accessing unstructured populations and establishing a chain of trust from surveyor to participants, it is also known to introduce biases. Indeed, butchers with more social contacts are more likely to be recruited. However, as pigs get randomly distributed from their origin to the butchers, through livestock markets and middlemen, we expect that the origin and breed of the pigs will be the main source of variation, and not the slaughter slab where they eventually get slaughtered. Furthermore, by starting several "snowballs" in different types of slaughter slabs (i.e., different size, skill level, and social strata), this method ensured the recruitment of a large number of participants with diverse profiles, reducing the risk of bias in our sampling strategy.

\section{Conclusion}

The results of our study may complement the scarce public health data on pork-borne parasitic zoonoses in Nepal toward a better understanding of their relative impact and importance. In line with the low Trichinella infection prevalence in pigs, no outbreaks of human trichinellosis have been documented in Nepal to date. Recently, however, multiple human trichinellosis outbreaks have been reported from the north Indian state of Uttarakhand, bordering West Nepal, confirming the regional presence of this parasite (Sethi et al., in press). The burden of T. solium neurocysticercosis in Nepal remains poorly understood, but is likely significant, as neurocysticercosis prevalences of up to $47 \%$ have been documented in Nepalese seizure patients (Rajbhandari 2003). Finally, toxoplasmosis is known to occur in the Nepalese population at seroprevalences of nearly $50 \%$, and congenital toxoplasmosis cases are observed (Rai et al. 2011). A quantification of its actual public health importance, however, remains lacking.

Given the clear relevance of pork as a possible source of human illness in Nepal, further efforts should be undertaken to monitor the related animal and human disease burdens and to strengthen food safety throughout the pork production chain.

\section{Acknowledgments}

We would like to thank Ms. Anke Van Hul and Ms. Famke Jansen from the Institute of Tropical Medicine, Antwerp, Belgium, for their technical support during this study. The collaborating butchers are acknowledged for their helpfulness and cooperation. This study was part of a larger project supported by the Flemish Interuniversity Council, Belgium. This organization had no role in designing the study, analyzing the data, nor in writing or approving the manuscript.

\section{Author Disclosure Statement}

The authors declare no competing financial or other interests.

\section{References}

Datt Bhatt K. Seroprevalence of Toxoplasma gondii in pregnant women and slaughtered pigs of Chitwan district. Rampur, Nepal: BSc thesis, Institute of Agriculture and Animal Science, Tribhuvan University, 2012.

De Craeye S, Francart A, Chabauty J, De Vriendt V, et al. Prevalence of Toxoplasma gondii infection in Belgian house cats. Vet Parasitol 2008; 157:128-132.

Deckers N, Dorny P. Immunodiagnosis of Taenia solium taeniosis/cysticercosis. Trends Parasitol 2010; 26:137-144.

Desmonts G, Remington JS. Direct agglutination test for diagnosis of Toxoplasma infection: Method for increasing sensitivity and specificity. J Clin Microbiol 1980; 11:562-568.

Devleesschauwer B, Aryal A, Joshi DD, Rijal S, et al. Epidemiology of Taenia solium in Nepal: Is it influenced by the social characteristics of the population and the presence of Taenia asiatica? Trop Med Int Health 2012; 17:1019-1022.

Devleesschauwer B, Aryal A, Tharmalingam J, Joshi DD, et al. Complexities in using sentinel pigs to study Taenia solium transmission dynamics under field conditions. Vet Parasitol 2013; 193:172-178.

DLS. Livestock Statistics of Nepal, 2010/2011. Hariharbhawan, Lalitpur, Nepal: Government of Nepal, Ministry of Agriculture Development, Department of Livestock Services (DLS), 2011.

Dorny P, Phiri IK, Vercruysse J, Gabriel S, et al. A Bayesian approach for estimating values for prevalence and diagnostic test characteristics of porcine cysticercosis. Int J Parasitol 2004; 34:569-576.

Dorny P, Praet N, Deckers N, Gabriel S. Emerging food-borne parasites. Vet Parasitol 2009; 163:196-206.

FAO. Market-led Quality Meat Production and Processing. Pulchowk, Nepal: Food and Agriculture Organization of the United Nations (FAO), 2010.

FAO/UNDP. Nepal agricultural policy and strategies for poverty alleviation and food security, NEP/99/023:SPPD Report, Annex 1. Kathmandu, Nepal: Food and Agriculture Organization of the United Nations (FAO) and United Nations Development Programme (UNDP), 2003.

Frey A, Di Canzio J, Zurakowski D. A statistically defined endpoint titer determination method for immunoassays. J Immunol Methods 1998; 221:35-41.

Gómez-Morales MA, Ludovisi A, Amati M, Cherchi S, et al. Validation of an enzyme-linked immunosorbent assay for diagnosis of human trichinellosis. Clin Vacc Immunol 2008; 15:1723-1729.

Gómez-Morales MA, Ludovisi A, Amati M, Blaga R, et al. A distinctive Western blot pattern to recognize Trichinella infections in humans and pigs. Int J Parasitol 2012; 42:10171023.

Joshi DD, Poudyal MP, Jimba M, Mishra PN, et al. Epidemiological status of Taenia/cysticercosis in pigs and human in Nepal. J Inst Med 2001; 23:1-12. 
Joshi DD, Maharjan M, Johansen MV, Willingham AL, et al. Improving meat inspection and control in resource-poor communities: the Nepal example. Acta Trop 2003; 87:119-127.

Joshi DD, Moller LN, Maharjan M, Kapel CM. Serological evidence of trichinellosis in local pigs of Nepal. Vet Parasitol 2005; 132:155-157.

Karn SK, Hörchner F, Srikitjakarn L, Baumann M, et al. Crosssectional study of Trichinella spp in pigs in CDR, Nepal using pepsin digestion and ELISA serology. Southeast Asian J Trop Med Public Health 2008; 39:795-799.

Kayastha KP. A scenario on pig production in Nepal. In: Proceedings of a Workshop on Present Situation Challenges in Treatment and Elimination of Taeniasis/Neurocysticercosis in Nepal, December 7-9, 2005. Kathmandu, Nepal: National Zoonoses and Food Hygiene Research Centre, 2006:47-54.

Lunn D, Spiegelhalter D, Thomas A, Best N. The BUGS project: Evolution, critique and future directions. Stat Med 2009; 28:3049-3067.

Macdonald P, Du J. mixdist: Finite Mixture Distribution Models. R package version 0.5-4. 2011. http://CRAN.R-project.org/ package $=$ mixdist

Opsteegh M, Teunis P, Mensink M, Züchner L, et al. Evaluation of ELISA test characteristics and estimation of Toxoplasma gondii seroprevalence in Dutch sheep using mixture models. Prev Vet Med 2010: 96;232-240.

Praet N, Rodriguez-Hidalgo R, Speybroeck N, Ahounou S, et al. Infection with versus exposure to Taenia solium: What do serological test results tell us? Am J Trop Med Hyg 2010; 83:413-415.

R Development Core Team. R: A language and environment for statistical computing. R Foundation for Statistical Computing, Vienna, Austria. 2012. ISBN 3-900051-07-0, URL http:// www.R-project.org

Rai SK, Kubo T, Yano K, Shibata H, et al. Seroprevalence of Toxoplasma gondii infection in common meat animals and its public health importance in Nepal. J Inst Med 1996; 18:55-60.

Rai SK, Sharma A, Shrestha RK, Pradhan P. First case of congenital toxoplasmosis from Nepal. Nepal Med Coll J 2011; $13 ; 64-66$.

Rajbhandari KS. Epilepsy in Nepal. Neurol J Southeast Asia 2003; 8:1-4.

Sapkota, BS, Hörchner F, Srikitjakarn L, Kyule MN, et al. Seroprevalence of Trichinella in slaughter pigs in Kathmandu
Valley, Nepal. Southeast Asian J Trop Med Public Health 2006; 37:1078-1082.

Sethi B, Butola KS, Kumar Y, Mishra JP. Multiple outbreaks of trichinellosis with high mortality rate. Trop Doct 2013; doi: 10.1258/td.2012.110335.

Sharma M. Socio-demographic factors of pig farmers associated in transmission of taeniosis/cysticercosis. J Inst Med 2006; 28:57-60.

Speybroeck N, Devleesschauwer B, Joseph L, Berkvens D. Misclassification errors in prevalence estimation: Bayesian handling with care. Int J Public Health 2012 2013; 58:791795.

Thi NV, De NV, Praet N, Claes L, et al. Seroprevalence of trichinellosis in domestic animals in northwestern Vietnam. Vet Parasitol 2013; 193:200-205.

Thomas A, O'Hara B, Ligges U, Sturtz S. Making BUGS Open. R News 2006; 6:12-17.

Tropp S, ed. Nepal Human Development Report 2009: State Transformation and Human Development. Pulchowk, Kathmandu, Nepal: United Nations Development Programme, 2009.

Vercammen F, Vervaeke M, Dorny P, Brandt J, et al. Survey for Trichinella spp. in red foxes (Vulpes vulpes) in Belgium. Vet Parasitol 2002; 103:83-88.

Wilmaerts L. Prevalence of Toxoplasma gondii, Trichinella spiralis, and Taenia solium in pigs on two commercial farms in the Democratic Republic of Congo. Antwerp, Belgium: MSc thesis, Department of Animal Health, Institute of Tropical Medicine, 2010.

Yera H, Andiva S, Perret C, Limonne D, et al. Development and evaluation of a Western blot kit for diagnosis of human trichinellosis. Clin Diagn Lab Immunol 2003; 10:793-796.

Address correspondence to: Brecht Devleesschauwer Department of Virology, Parasitology and Immunology Faculty of Veterinary Medicine Ghent University Salisburylaan 133 9820 Merelbeke Belgium

E-mail: brecht.devleesschauwer@ugent.be 